\title{
Citation:
}

Stylianides, G. J., Sandefur, J., \& Watson, A. (in press). Conditions for proving by mathematical induction to be explanatory. Journal of Mathematical Behavior. 


\begin{abstract}
In this paper we consider proving to be the activity in search for a proof, whereby proof is the final product of this activity that meets certain criteria. Although there has been considerable research attention on the functions of proof (e.g., explanation), there has been less explicit attention in the literature on those same functions arising in the proving process. Our aim is to identify conditions for proving by mathematical induction to be explanatory for the prover. To identify such conditions, we analyze videos of undergraduate mathematics students working on specially designed problems. Specifically, we examine the role played by: the problem formulation, students' experience with the utility of examples in proving, and students' ability to recognize and apply mathematical induction as an appropriate method in their explorations. We conclude that particular combinations of these aspects make it more likely that proving by induction will be explanatory for the prover.
\end{abstract}

Key words: College/university mathematics; Examples; Explanation; Proof by mathematical induction; Problem design; Proving 


\section{CONDITIONS FOR PROVING BY MATHEMATICAL INDUCTION TO BE EXPLANATORY}

\section{Introduction}

Although there has been considerable research attention on the functions of proof (explanation, verification, generation of new knowledge, etc.), there has been less explicit attention in the literature (to the best of our knowledge) on the same functions arising in the proving process. In relation to proof, there are also different perspectives in the literature about the various functions proof can serve. One perspective is to consider these functions as the purposes proof can serve for the prover or the reader of the proof (we call this the subjective perspective), whereas an alternative perspective is to consider the functions as characteristics of the text of the proof (we call this the absolutist perspective). There are also hybrid perspectives that combine aspects of these two perspectives.

The terms 'proof' and 'proving' have also been used in a number of different ways in the literature. It is beyond the scope of this paper to discuss the different meanings of 'proof' and 'proving' (see Stylianides, Stylianides, and Weber [2016] for a review). It is important, though, to clarify our use of these terms herein. We consider proving to be the activity in search for a proof (e.g., Stylianides, 2007, p. 290), whereby proof is the final product of the proving activity that meets the following criteria: it is "a valid argument based on accepted truths for or against a mathematical claim that makes explicit reference to 'key' accepted truths that it uses" (Stylianides, 2009, p. 265). Again, it is not necessary for our purposes to unpack all the terms in the definition of proof (the reader can refer to Stylianides [2009] for elaboration). We clarify, though, that the term 
'accepted truths' is used broadly to include the axioms, theorems, definitions, and statements that a particular community may take as shared at a given time. Which accepted truths are 'key' and, thus, should be explicitly referenced in a proof depends on the audience of the proof (for example, some accepted truths may be considered trivial or basic knowledge for a particular audience and thus may be omitted from a proof). It is also important to note that our definition of 'proving' implies that this activity can include a cluster of other related activities that are often precursors to producing a proof such as testing examples to generalize and formulate conjectures, testing the conjectures against new evidence and revising the conjectures to conform with the evidence, and providing informal arguments that show the viability of the conjectures.

In this paper we adopt the subjective perspective we described above and we investigate conditions for proving by mathematical induction to be explanatory for the prover (or provers in the case of collaborative activity) as opposed to be explanatory for the reader (or readers) of the proof. Our adoption of the subjective perspective does not suggest that we consider it to be better than the absolutist perspective. We adopted the subjective perspective because of the particular focus of our study: we are interested in identifying conditions for proving by mathematical induction to be explanatory for university students working in small groups on proof problems. We say 'proving' as opposed to 'proof' because, contrary to most prior research on the functions of proof, we are not focusing on the final product of the proving activity (i.e., the proof) to examine whether that was explanatory for the provers. Rather, we focus 
on the proving activity that leads to the use of a particular method to develop a proof for a mathematical statement, with particular attention to provers' use of mathematical induction. In the next section we elaborate on the scope of the paper, beginning with a discussion of our notion of 'proving activity that is explanatory for provers' (or 'explanatory proving' for short).

\section{Elaboration on the Scope}

To define our notion of explanatory proving, which is new in this paper, we will seek to maintain consistency with the way prior research defined proof to be explanatory for the prover (or provers), namely, whether the proof illuminated or provided insight to a prover into why a mathematical statement is true (Bell, 1976; de Villiers, 1999; Hanna, 1990; Steiner, 1978) or false (Stylianides, 2009). We will thus consider the proving activity to be explanatory for the prover (or provers) if the method used in a proof provided a way for the prover to formalize the thinking that preceded and that illuminated or provided insight to the prover into why a statement is true or false.

This definition of explanatory proving is not specific to mathematical induction, but could apply to any proof method. Given our focus here on mathematical induction, however, we offer an example of how proving by mathematical induction could be explanatory for provers: provers could use recursive reasoning (that is, reasoning relating to or involving the repeated application of a rule or procedure to successive results) in their exploration of a mathematical statement in ways that could help provers 
see informally the structure of the inductive step in a possible proof by induction; the provers could subsequently apply mathematical induction to formalize their thinking and verify the truth of the statement.

Implied in our definition of explanatory proving is the idea that the proof followed naturally from the provers' investigation of a mathematical statement. This continuity between the final product (the proof) and the earlier parts of the proving process (the exploratory phase of proving) has similarities to the notion of cognitive unity. This notion derived from a long-term teaching experiment in Italy (e.g., Boero, Garuti, Lemut, \& Mariotti, 1996a; Boero, Garuti, \& Mariotti, 1996b; Garuti, Boero, \& Lemut, 1998) that aimed to introduce school students to Geometry and that focused on engaging students with problems that required both the development of a conjecture and its proof. The notion of cognitive unity was described and used in a number of different ways over the years (see Mariotti [2006, pp. 182-184] for a discussion). Below is one of the earliest and most commonly cited descriptions (Boero et al., 1996a, p. 113; the original was in italics):

- during the production of the conjecture, the student progressively works out his/her statement through an intense argumentative activity functionally intermingling with the justification of the plausibility of his/her choices; - during the subsequent statement proving stage, the student links up with this process in a coherent way, organising some of the justifications ("arguments") produced during the construction of the statement according to a logical chain.

As indicated in the previous excerpt, the notion of cognitive unity captures a possible continuity between the arguments that students produced to support or reject a specific conjecture and the final proof for the conjecture (Mariotti, 2006). The continuity in cognitive unity is similar to the continuity we described earlier in our 
definition of explanatory proving since, in both cases, there is a connection between the proof and parts of the proving process that preceded the proof's development. Yet the continuity in cognitive unity is more restricted than the one in our definition of explanatory proving. In cognitive unity the continuity is focused on the arguments that led to a conjecture and its proof. This is only one example of the kind of continuity we could have in our notion of explanatory proving. In explanatory proving, the provers could develop an insight (cf. the notion of 'conceptual insight' in section 4.2) into the truth of a conjecture not necessarily because of an argument they developed, but because of recognizing a 'familiar territory' based on a particular representation (or 'appropriation of the statement' in Garuti et al.'s [1998] terms) that led them to see the relevance or usefulness of a particular proof method in turning their insight into an acceptable proof (cf. the notion of 'technical handle' in section 4.2).

We decided to focus in this paper on mathematical induction not only because of its importance in the discipline of mathematics, but also because it is a proof method that is known to provide particular difficulties for students in achieving cognitive unity (Mariotti, 2006, pp. 187-188) and is frequently viewed by students as a proof that verifies without necessarily explaining. Regarding the latter, in a study of university students taking an introduction to proof course, Smith (2006) found that some students did not view mathematical induction as explanatory, but "as an algorithm they can apply almost blindly" (pp. 80-81). In fact, one of the four students in Smith's study was uncomfortable with induction as her approach to constructing proofs was to look for "arguments that explain why processes work" (p. 81; emphasis in original). Dubinsky 
(1986) also expressed doubts about the subjective explanatory value of induction for undergraduate mathematics students.

Formal mathematical induction arguments are not typically considered as important to the school mathematics curriculum as they are to the university mathematics curriculum. However, the kind of reasoning embedded in proof by mathematical induction appears in different ways in the school curriculum such as in sequential reasoning tasks in the lower secondary school years, which anticipate the central role of recursive reasoning in numerical and iterative methods taught in the upper secondary school years. Research also supports the idea that even young students can produce rudimentary versions of mathematical induction and engage in relevant activities (Maher \& Martino, 1996; Reid, 2002) even though the term 'mathematical induction' is typically not used with those students. In several countries a formal introduction to proof by induction takes place within the secondary school curriculum, so teachers need to have good knowledge of this proof method (Movshovitz-Hadar, 1993; Stylianides, Stylianides, \& Philippou, 2007).

The rest of the paper is organized as follows. In section 3 we discuss literature related to the explanation function of proof in general but also of mathematical induction in particular (as we mentioned earlier, prior research focused on the functions of proof rather than those of proving). This discussion also addresses the two perspectives on the functions of proof we mentioned earlier. In section 4 we describe the framework we used in this paper. As we explain, the framework served multiple purposes in our study. We conclude this section by connecting the framework with our 
meaning of 'explanatory proving,' summarized previously. In section 5 we discuss

studies about university students' understanding of proof by mathematical induction.

This discussion provides a useful backdrop for our analysis of the videos of students'

work on the proof problems. In section 6 we discuss our methods, including providing a

description of the course from which we derived our data. As we explain, the students in

our study were taking a course that incorporated particular features that we

hypothesized would support situations in which students' proving activity would involve using mathematical induction in an explanatory way. In sections 7 through 9 we apply the framework to analyze the two problems we used for this paper and the students' work on those problems. Finally, in section 10 we summarize and further discuss our findings.

\section{Explanation Function of Proof}

Historically, there has been a debate on what it means to say that a proof in mathematics explains. Steiner (1978) argued that "an explanatory proof makes reference to a characterizing property of an entity or structure mentioned in the theorem, such that from the proof it is evident that the result depends on the property" (p. 143). Steiner defined 'characterizing property' as a property whose absence would cause the proof to fail and also one that could be replaced by other characterizing properties to produce new theorems. As Weber (2010, p. 33) pointed out, though, few researchers who used Steiner's definition in mathematics education applied the latter feature of characteristic property. Taking Steiner's definition further, Hanna (1990) used 
"the term explain only when the proof reveals and makes use of the mathematical ideas which motivate it" (p. 10; emphasis in original). She gave the example that the induction proof for the following formula

$$
1+2+\cdots+n=\frac{n(n+1)}{2}
$$

is a proof that demonstrates (verifies), whereas a proof that would involve adding $1+2+\ldots+n$ to $n+(n-1)+\cdots+1$ and dividing by 2 would be a proof that explains the same result. Hanna (2000) sees explanatory power as a property of a proof, possibly in an educational context, claiming that the educational value of proof is to enable understanding. However, this leaves us with the problem of defining 'understanding.'

For the most part, the debate on whether a proof is explanatory hinges on one's use of the term 'explain.' The research we discussed in the previous paragraph seems to consider explanation as a property of the proof, which relates more to the absolutist perspective we mentioned at the outset of the paper, so that its explanatory power can be identified by analysis of the text of the proof. Professional mathematicians tend to talk of the explanatory power of a proof in terms of whether it provides new insights into the field of application, new ways of reasoning about particular objects, or new connections between fields of study (e.g. Hersh, 1993; Weber, 2010). Kitcher (1989), focusing on the role played by proof in advancing mathematics, describes explanation as a chain of reasoning based on 'relevance relations' and mathematical truths and ends with a statement describing a phenomenon. In other words, for Kitcher an explanation involves reasoning that provides a causal answer to a why question for the class under consideration. In relation to proof by mathematical induction, Lange (2009) used a 
purely logical argument that proof by induction is never explanatory based on his definition that, for a proof to be explanatory, it must follow from other mathematical truths and the argument must not be circular. In particular, Lange argues that you cannot use $P(1)$ to show $P(k)$ and also use $P(k)$ to show $P(1)$. According to Lange "[t]his argument does not show that some proofs by mathematical induction are not explanatory. It shows that none are..." (p. 209).

Kitcher's (1989) theory of explanation, that is often used in juxtaposition to that of Steiner (1978), grew from his work in the philosophy of science, in which there is often a phenomenon that can be described, exemplified, and verified in a variety of ways. Lange's (2009) exposition refers to no phenomena outside the structure of the symbolic argument itself. Taking these two approaches together suggests that an element of explanatory power is that the mathematical phenomenon being proved can somehow be manifested in another way at some stage of the proof. This avoids what some authors call the symmetry of ordinary mathematical induction proofs which appear to be only self-referential (Cariani, 2011).

Our context is educational, in that we are interested in what students do when learning to prove, particularly in whether proving by mathematical induction has explanatory power for the provers about the relations being proved. Returning to Kitcher (1989) we therefore question whether 'relations' that might appear 'relevant' for novice mathematicians, would also be relations whose relevance might support advances in the field. Clearly, students would not find as explanatory a proof that involved relations about which they were ignorant, so arguments that detach 
explanatory power from the prover are not generally helpful for us except to draw attention to the need to draw on other representations of mathematical phenomena than those given.

We are helped by a number of authors who agree that an explanatory proof answers 'the why questions' (Hanna, 2000; Hersh, 1993), but this begs the question 'whose why questions?' We thus consider explanatory power to be context-dependent (Resnick \& Kushner, 1987), which is why we explore students' proving processes in a detailed analysis of videos of their work. The explanatory power of proving by mathematical induction can help students develop their understanding of the mathematical ideas involved (ideas of number theory in our study), ideas about proof, or both. There are two places where alternative representations can introduce explanatory power to proving by induction: the base step (establishing an initial case) and the inductive step (proving the implication $P(n) \Rightarrow P(n+1)$ ). We are looking for ways in which students engage with these in ways that give them insight based on their knowledge and experience. In other words, we are looking for moments where the proving process has reduced the situation to something 'familiar' (Weber, 2010, p. 34) for the students. This is the opposite of Weber's (2010) description of whether a proof is explanatory for a reader (p. 34): that a proof is explanatory if a reader can translate a formal argument into a less formal argument in a different semantic representation system.

Weber's (2010) student-centered definition of explanatory proof, similar to Harel and Sowder's $(1998,2007)$ framework of proof schemes, relate more to the subjective 
perspective, which is also the perspective we adopt in our study. Harel and Sowder $(1998,2007)$ have used a student-centered view in their proof schemes framework and highlighted the subjective sense in which the terms in their framework should be interpreted. This stance is reflected in the definitions they offered for 'proving' and its two subprocesses, 'ascertaining' and 'persuading.' For Harel and Sowder (2007, p. 808), proving "is the process employed by an individual (or a community) to remove doubts about the truth of an assertion," ascertaining "is the process an individual (or a community) employs to remove her or his (or its) own doubts about the truth of an assertion," whereas persuading "is the process an individual or a community employs to remove others' doubts about the truth of an assertion." As the previous definitions suggest, Harel and Sowder's proof schemes focus more on conviction, that is, the 'verification' (e.g., Bell, 1976; de Villiers, 1999) function of proof, rather than the explanation function. Harel and Sowder (2007) noted that, for a student to engage in mathematics as sense-making, the student should not only ascertain oneself that a topic/procedure makes sense, but he or she "should also be able to convince others through explanation and justification of her or his conclusions" (p. 809). 'Sense-making' is clearly at the core of the explanatory function of proof, but describing 'explanation' simply as 'sense-making' would not be that useful for our analysis in this paper.

The subjective perspective in Harel and Sowder's proof schemes framework also informed Harel's (2001) study in which he taught mathematical induction in a number theory course for prospective secondary teachers. The focus of our study on conditions for proving by mathematical induction to be explanatory for students has some 
similarities to what Harel (2001) called process pattern generalization, in which students focus on generalizing the regularity in the process being performed and not just the regularity in the observed result/pattern. Mariotti (2006, pp. 187-188) connected the notion of process pattern generalization with that of cognitive unity, suggesting that it is more likely to achieve cognitive unity in proof by mathematical induction when students generate conjectures with process pattern generalization. While there is a connection between process pattern generalization and our notion of proving that is explanatory for students, there is also a subtle difference. In process pattern generalization the students focus on why statement $P(n-1)$ implies statement $P(n)$ (the process), while we are concerned with students' proving activity which explains for them why a statement is true or false.

More broadly, there are three important differences between our study and that of Harel (2001). One difference is that the problems Harel used were implicitly recursive in nature, that is, they were stated as "show something about statement $n$. " The problems we used in our study were not implicitly recursive: they were posed as "make a conjecture about the conditions under which a statement $P(n)$ is true or false," where it would likely appear to the student as if the statement could be true for one $n$-value but not the next. We consider statements in this latter form as particularly rich as they are less obviously conducive to a proof by induction - the statement to be proved is not given in the problem. The students in our study therefore had to undertake some justified mathematical exploration to arrive at a statement they needed to prove (similar to the explorations students had to undertake in the studies by Boero et al. 
[1996a, 1996b] and Garuti et al. [1998]), in the form of a conjecture, and decide whether to use mathematical induction, drawing this method from their repertoire of proof methods. A second difference between our study and Harel's (2001) is that he used process pattern generalization as a means for teaching students mathematical induction, while the students in our study already knew about induction and so the problems provided an opportunity for our students to select and use induction as an appropriate method to tackle a particular proof problem. A third difference is that Harel was interested in students using the inductive step as a means to explain why induction was useful in constructing proofs, while we were interested in studying whether and how students used induction to explain why a statement is true or false.

\section{Framework}

This section is organized into two parts. In the first we present the framework we used in this paper. In the second we connect the framework with the meaning of 'explanatory proving' as summarized in section 2.

\subsection{Framework Description}

The framework served multiple purposes in our study. First, the framework consists of four aspects that we hypothesized could support students' engagement in situations in which proving by mathematical induction could serve an explanatory function for them. Second, the framework had informed the redesign of an Introduction to Proof course for undergraduate mathematics students taught by the second author, which is the course from which we draw our data (see section 6), and hence has 
ecological validity. Third, we used the framework to analyze the two problems we focus on in this paper and the students' work on those problems (see sections 7 through 9).

The framework is an adapted version of a framework that we proposed previously (Sandefur, Mason, Stylianides, \& Watson, 2013, p. 337), which was derived from the literature and our analysis of a different subset of videos from earlier versions of the Introduction to Proof course. Although the focus of our previous analysis was on aspects of situations in which example generation can play a constructive role in the proving process, we hypothesized that similar aspects could facilitate, and provide an appropriate lens for our analysis of, students' possible use of mathematical induction in an explanatory way. This is because mathematical induction often involves the generation of sequential examples which can be analyzed to find recursive patterns that might then be generalized.

Below we describe the four aspects of the framework, which conjoins qualities of students and of problems. We also explain how these aspects may facilitate students' engagement in situations in which proving by mathematical induction could serve an explanatory function for students.

Firstly, a quality of problems:

(1) Problem formulation. We know from our earlier work, but also from prior research such as studies on cognitive unity (e.g., Garuti et al., 1998), that the formulation of proof problems influences the ways in which students approach the exploratory phase of their work; a problem that more or less directs students to use a certain method and gives a manipulable representation is less likely to 
require students to engage with conceptual meaning. The problems we focus on for this study were phrased so that they did not indicate the use of a particular proof method for solution; mathematical induction was relevant though not necessary for the solution of the problems. The problems were intentionally phrased so that students would need to do some exploratory work in order to identify an appropriate statement to prove and solve the problem. We hypothesized that the students' initial exploratory work is likely to involve example generation, which might lead to recursive reasoning to explain why a statement was true, before the students use proof by induction to formalize their thinking and verify the truth of the statement. In other words, we hypothesized that the need for these explorations would make it unlikely that students would resort to an instant and rote application of the induction method which, as we will see in our review in section 5 , is common for students especially when induction is the obvious or expected method.

Secondly, two qualities of students:

(2) Experience with utility of examples in proving. This relates to students' experience of the possible utility of constructing examples when proving, and students' familiarity with how constructing examples can be used not just to verify but also to expose structural relationships and generate conjectures. Students who are familiar with the use of examples to explore are more likely to generate examples during an exploratory phase of proving. This quality can be particularly relevant in situations where the problem is formulated, as we 
described in aspect 1, in a way in which it is not clear what would be an appropriate statement to prove in order to solve the problem.

(3) Personal example spaces and technical tools. The example spaces on which students draw need to be sufficiently complex and robust so as to support the display of underlying structural relationships and to enable these to be manipulated. This quality also includes students' familiarity with and ability in using appropriate technical tools (such as the proof method of mathematical induction), including students' ability to recognize the possible use of these tools in unfamiliar situations.

Finally, a conjunction of qualities of students and the problem:

(4) Relational necessity. We use a notion of 'relational necessity' to address whether a combination of a problem and students' knowledge, experience, and technical capability are such that the proof can be completed by manipulation without paying attention to underlying mathematical relations (Sandefur et al., 2013, p. 337), or whether it is necessary for them to think about the relations. The problem formulation characteristics we described in aspect 1 suggest that students (independently of the level of their knowledge, experience, and technical capability) cannot complete a proof by mere manipulation, without exploration or paying attention to underlying mathematical relations. Exploration activity can be reinforced when a problem is formulated as in aspect 1 above (e.g., phrased as "make a conjecture and then prove your conjecture"). Building on Raman (2003), we also suggested in Sandefur et al. (2013) that, to achieve a proof where manipulating 
given symbols is not enough without paying attention to underlying mathematical relations, it is often necessary for provers to align their conceptual insight (i.e., a sense of a structural relationship pertinent to the phenomenon of interest that indicates why the statement is likely to be true or false) with a technical handle (i.e., a way of manipulating or making use of the structural relations that support the conversion of a conceptual insight into a proof). ${ }^{1}$

\subsection{Connection between the Framework and Explanatory Proving}

In section 2 we defined the proving activity to be explanatory for the prover (or provers) if the method used in a proof provided a way for the prover to formalize the thinking that preceded and that illuminated or provided insight to the prover into why a statement is true or false. So, in our study, we are not focused on the final proof, but on whether the proving activity is explanatory. To understand if a proving activity is explanatory for a group of students, we focus on the process by which the provers arrived at not only a particular approach or technical handle to writing the proof of a statement, but also the development of a conceptual insight which resulted in an understanding of why the statement was true or false. The proving activity can be explanatory for the provers even if they are not able to produce a complete and polished proof at the end.

\footnotetext{
1 We clarify that the definitions of 'conceptual insight' and 'technical handle' in parentheses are a loose paraphrase of Raman's (2003) definitions of 'key idea' and 'technical handle.' Also, whereas for Raman these notions are absolute features of the proof, we are using them as components of subjective experience.
} 
In our study, students were given problems of the form "make a conjecture about what is true in this situation, then prove your conjecture" (a strong version of aspect 1 of our framework). This problem formulation necessarily leads to the relational necessity (aspect 4) of exploring, usually through examples, to determine a conjecture. Once a conjecture is made, one of several things can happen, two of which frequently occur. One is that the students, possibly having little insight into why the conjecture is true, can still proceed to develop a proof. The second, which is a stronger version of relational necessity, is that the students continue to explore, usually through the continued use of examples, until they have developed some conceptual insight into why their conjecture is true; then, with the use of a technical handle tied to their conceptual insight, students can end up with a proving experience which is explanatory for them. In our framework, we hypothesized that the second possibility might be enhanced for students who meet aspects 2 and 3, as the students in our study did.

The idea of explanatory proving, as we just connected it to aspects of our framework, can be seen also in some examples from the literature. Maher, Muter, and Kiczek (2007) discuss an episode in which tenth-grade students work on the problem of finding how many pizzas can be formed if there are 5 toppings to choose from. After attempting to count the number of pizzas, which they gave as 31 , one student connected each possible pizza with each possible binary number with 5 digits, counting ' 0 ' when a toppings was not included and ' 1 ' when a topping was included. The proof was explanatory for others as to why 32 is the correct answer, not 31 . In addition, the process of developing this proof was also explanatory for the prover as he made 
connections between binary numbers and ingredients, resulting in conceptual insight and technical handle which were inexorably intertwined. We see also this idea of explanatory proving in Hanna's (1990) description of the proof of the statement that the sum of the first $n$ positive integers equals $n(n+1) / 2$, where the integers are added twice by reversing the order. The manipulation of reversing and adding the sum twice leads to both a conceptual insight into why the sum equals the expression and a technical handle that allows the prover to verify this result.

\section{Students' Understanding of Proof by Mathematical Induction}

The discussion in this section shows that many university students seem to be convinced by superficial features of proof by mathematical induction (e.g., Dubinsky, 1986, 1990; Dubinsky \& Lewin, 1986; Harel, 2001; Movshovitz-Hadar, 1993; Stylianides et al., 2007). A common problem is students' limited understanding of the necessity and utility of both the base step and the inductive step. This could go some way towards explaining why students frequently view induction merely as proof that verifies and not also as proof that can also explain.

As well as the difficulties just described, Knuth (2002) found that a cohort of inservice US secondary mathematics teachers accepted a proof by mathematical induction not because they understood the method, but merely because they knew the method could be used technically in similar mathematical situations (these situations would not meet aspect 1 of our framework). It would be unlikely therefore for proving by induction to serve an explanatory function for these teachers. The same would apply 
for many of the 95 Cypriot senior undergraduate students in the Stylianides et al. (2007) study, which showed additional difficulties centred around: the meaning of the inductive step in proving the implication $P(n) \Rightarrow P(n+1)$ for an arbitrary $n$ in the domain of discourse of $P(n)$; and the possibility that a statement proved by mathematical induction might apply to an extended domain beyond the one proved.

These difficulties relate and add to those identified by Dubinsky $(1986,1990)$. Specifically, in a study with sophomore students (mainly computer science and engineering majors), Dubinsky identified difficulties with: (1) the character of the implication as a total entity (instead of trying to prove the inductive step $P(n) \Rightarrow P(n+1)$, several students tried to prove $P(n+1))$; and (2) the essence of the base step, which was viewed by some students as a procedure without any real meaning. Similarly, in a study with 22 mathematics majors, Dubinsky and Lewin (1986) found that students had difficulties in encapsulating modus ponens and in coordinating it with the structure of implication-valued functions.

Several studies followed the idea of teaching proof by mathematical induction using situations that require recursive reasoning in order to avoid creating an impression that this proof method is essentially about manipulating symbols. Ron and Dreyfus (2004) used models, such as Tower of Hanoi, to provide a meaning for the base and the inductive steps. Harel (2001) introduced induction to a number theory class for prospective secondary teachers through "engagement with implicit recursive problems," also using the Tower of Hanoi problem, in which the students developed quasi-inductive arguments; these are informal arguments that depend on the patterns, 
either of ascent (from simpler cases to more complex cases) or descent (from complex cases to simpler cases), observed in examples. Davis, Grassl, Hauk et al. (2009) introduced a necessity-based approach in which students had to overcome various problem-specific obstacles to develop such quasi-inductive arguments. Brown (2008), in a study with both college and high achieving sixth-grade students, used problems promoting process pattern generalization. This resulted in students generating quasiinductive arguments and developing their understanding of the implications of various infinite processes.

In all approaches described in the previous paragraph students encountered situations in which recursive reasoning arose naturally through exploration as a partial explanation, with some of these situations being introduced before students formally learned about proof by induction and others being used to deepen students' understanding of induction. As we mentioned earlier, the students in the videos we analyze in this paper already knew about proof by induction and we were interested to see whether and how they could use this knowledge in proving situations in which induction was one of several possible methods to approach a problem. This, in turn, would help us identify and characterize those situations that provided a space within which students voluntarily used proving by induction in an explanatory way.

\section{Method}

\subsection{Context and Data}


Since 2003, Sandefur has been teaching an Introduction to Proof course to undergraduate mathematics students at a private American university. Students in this course will have taken two semesters of Calculus and, possibly, Multivariable Calculus and/or Linear Algebra. This course introduces students to a variety of proof methods including direct, contraposition, cases, contradiction, and induction. The introduction to mathematical induction had elements of what Harel (2001) calls "standard," that is, giving students the method of mathematical induction and having them work a number of typical induction problems, such as verifying the formula for a finite geometric series, followed by a few problems that intrinsically require recursive reasoning (problems in which process pattern generalization leads naturally to the induction step).

As a result of work done jointly with the other two authors of this paper and John Mason, and previous work done with Raman, Sandefur, Birky, Campbell, and Somers $(2009)^{2}$, Sandefur had modified this course to align more consistently with the four aspects of the framework we discussed in section 4. In particular, students were rarely given a statement to prove, but instead were usually given statements to prove true or false in which an appropriate proof method was generally not obvious from the statement. For example, students might be given a function such as

$$
f(x)=\left\{\begin{array}{rr}
x+1, & x<0 \\
2 x+a, & x \geq 0
\end{array}\right.
$$

\footnotetext{
2 This work has been supported by the following National Science Foundation grant (number 1020161): Research Based Videos for Developing Mathematical Thinking Skills in Proof Writing and Problem Solving.
} 
and be asked to prove whether or not it is one-to-one and/or onto. Note that if $a=1$ then $f$ is one-to-one and onto, if $a=0$ then the function is onto but not one-to-one, and if $a=2$ then the function is one-to-one but not onto. The idea was to give students problems which looked similar but that had different results so students would be unable to guess the truth of a statement without further exploration (aspects 1 and 4 of the framework). This approach encouraged students to think deeply about a problem before deciding on what to prove and what proof method to use.

In their explorations, students were taught to look for conceptual insight into why a statement was true or not. This usually consisted of constructing examples and not looking only at if the examples verified the statement, but also why the examples satisfied the statement (aspects 2 and 3). Once they developed their conceptual insight, they learned to look for technical handles that would allow them to write a proof (aspect 4). This could include using both a proof method (contraposition, contradiction, etc.) and appropriate mathematical tools (such as finding a $\delta$ to go with an $\varepsilon$, or make proper use of a definition) necessary to develop the proof.

With this approach to proving, the students in the course were not only expected to develop their knowledge of and capability in using various proof methods (including mathematical induction), but also to develop their knowledge of the mathematical ideas involved (such as ideas about number theory in the videos we analyze later). As we mentioned in section 3, this dual possible benefit for students is part of what learners could gain from the explanatory power of proving. 
Near the end of one of the modified versions of the course we described earlier, Sandefur divided the class into groups of three, forming groups that were as homogeneous as possible in terms of their problem solving skills so that all three students in each trio might contribute. He then videotaped each group working proof problems. While all of these problems met aspect 1 of the framework, induction was relevant for only two of the problems even though it was neither implied nor necessary for solution. For this paper, we analyze the four videos that related to these two problems; the videos involved two trios of students working on Problem 1 and two trios working on Problem 2. Within these videos, there were elements in students' work that suggested that they voluntarily used recursive reasoning followed by formal application of mathematical induction as a way to formalize the thinking that preceded the proof in their exploration and provided insight to them into why a statement is true or false. Intervention was zero or minimal, but, when necessary, the instructor (Sandefur) would normally try to bring the session to an end under an hour.

We note that, by the time the videos were taken, the students were familiar with proof by mathematical induction and had enough knowledge that enabled them to recognize and apply this method when appropriate. Also, the students in the videos knew that they were expected to produce a proof, whether it was explicitly mentioned in the problem formulation or not, and they seemed to understand the requirements of proof, that is, they aimed for a "conventional justification scheme" (Stylianides \& Stylianides, 2009, p. 321).

\subsection{Analytic Approach}


We viewed each of the videos, separately and together, having extensive discussions about the ways in which the students decided to use, and how they ended up using, mathematical induction in their proving activity. In our analysis, we used the framework we presented in section 4. As we described in section 6.1, the students in our study had experiences in relation to all four aspects of the framework, which made them an appropriate sample for us to investigate our hypothesis that their engagement with new problems that satisfied aspect 1 of the framework would likely give rise to situations in which students' engagement with proving by mathematical induction would be explanatory for them.

\section{Problem 1: Analysis of the Problem and Discussion of Students' Work}

\subsection{Analysis of Problem 1}

Problem 1: Develop a conjecture about the remainder when $5^{n}$ is divided by 3 and prove your conjecture.

Problem 1 is consistent with aspect 1 of the framework in that it is not stated in a form that suggests to students the use of proof by mathematical induction. The expected conjecture is that the remainder is 1 when $n$ is even and 2 when $n$ is odd. A conceptual insight could be to note that $5^{2 n}=25^{n}$ and that $25 \equiv 1(\bmod 3) \Rightarrow 25^{n} \equiv$ $1^{n}(\bmod 3) \equiv 1(\bmod 3)$. Alternatively, given the students in the videos had not yet worked with modular arithmetic, a conceptual insight could be to note that the terms being multiplied are of the form $(3 k+2)$ and $(3 k+1)$ and all that is important in determining the remainder of the products is the product of the remainders for each term. A technical handle for constructing a proof could be to use induction on $n$ even with the case of $n$ 
odd being a corollary. Because this problem results in different conjectures for odd and even values of $n$, it does not obviously lend itself to a $P(n) \Rightarrow P(n+1)$ argument, making it hard for students to notice the possible usefulness of induction in its solution.

\subsection{First trio's work on Problem 1}

This trio of two males and a female - called lan, John, and Kate ${ }^{3}$ - made a table of values straight away from which they made the correct conjecture, and John said "so this is induction then?" very early on. Note that while the pattern has led them to the correct conjecture and a technical handle that would allow them to construct a proof, they had not yet developed conceptual insight into why this might be the case. After some thinking, they rewrote $5^{n}=5^{2 j}=\left(5^{2}\right)^{j}$ and $5^{2 j+1}=5\left(5^{2}\right)^{j}$. John pointed at the first of these and said: "We need to do this first and it has some remainder, then [pointing at the second term] multiplying by 5 changes the remainder." A few minutes later, lan, developing some conceptual insight, pointed to $\left(5^{2}\right)^{j}$ and said that " $5^{2}$ has a remainder of 1 and 1 to any power is 1." John said: "This is an induction, right?" He then continued: "If we proved it for the first one and assumed it for [pause] $5^{n}$, then we would [pause] we would have to prove it $5^{n+2}$ for each even case, and like the opposite for the odd cases. Does that make sense?" After that John said again: "That will prove by induction that all the evens have a remainder of 1 . Do you want to try doing that?" At this point, John seemed to have integrated conceptual insight with a technical handle, pointing the trio's proving activity in a correct path for constructing a proof. In the

\footnotetext{
${ }^{3}$ All student names in the paper are pseudonyms.
} 
following, the students worked on developing the details of an induction proof formalizing these ideas.

To get started on the proof, they wrote $\left(5^{2 j}\right)\left(5^{2}\right)$ and John said: "If the first term $\left(5^{2 j}\right)$ has a remainder of 1 and $5^{2}$ has a remainder of 1 , can we assume their product has a remainder of 1 ?" They thought a bit about this and then John said: "We have to divide by 3 somewhere in here." After being confused by thinking about the division by 3 , they thought for some time. They wrote $\left(5^{2 j}\right) / 3=3 i+1$ at one point, commenting that they had to divide by 3 somewhere. Later they even divided both

terms by 3 , writing $\frac{5^{2 j}}{3} \frac{5^{2}}{3}$. Then nearly ten minutes later, Kate wrote $(8(3)+1)^{j}$ and they stated that this must have a remainder of 1 but they did not know how to show it. They finally resolved their confusion with the division by 3 and wrote $\left(5^{2 j}\right)\left(5^{2}\right)=$ $(3 k+1)(3(8)+1)=3(25 k+8)+1$, realizing this completed the proof of the even case. This was about 30 minutes into their exploration. They then started to use induction for the odd case instead of multiplying the even case by 5 , but the instructor stopped them and suggested they could have used the even case to prove the odd case, which they did without difficulty.

\subsection{Second trio's work on Problem 1}

The second trio worked similarly to the first trio, eventually having the same conceptual insight as the first trio, writing $\left(5^{2 n}\right) 25=(3 k+1) 25=3(25 k+8)+1$. They similarly used mathematical induction, trying to consider the even and odd cases at once, and assuming that "for some $n, 5^{2 n}=3 k+1$ and $5^{2 n+1}=3 m+2$." The second trio had more difficulty than the first writing a syntactically correct proof by 
induction, possibly because of their many careless algebraic mistakes. For example, their assumption that $5^{2 j}$ has a remainder of 1 when divided by 3 was translated into $5^{2 j} / 3=$ $3 k+1$ and $5^{2 j+2} / 3=(3 k+1) 5^{2}=3 k+26$. Eventually one student removed the division by 3 and another student realized they had to distribute the $5^{2}$, resulting in $5^{2 j+2}=(3 k+1) 5^{2}=$ $3(25 k+8)+1$, at which point one student exclaims "whoa, that has a remainder of 1. " They went on to show the odd case had a remainder of 2 . They were not able to produce a correct proof by induction, because they neglected to make the correct induction assumption that " $5^{2 j}=(3 k+1)$ for some $j$ and $k$." Part of their difficulties to produce a proof may have been due to the fact that these students did not work in an organized manner, having work all over the board that made it hard for them to keep track of their thinking.

\subsection{Commentary on both trios' work on Problem 1}

The first trio considered induction at the beginning although they did not seem to know at that point why their conjecture was true or how to construct the proof. Writing the expression as $\left(5^{2}\right)^{j}$ and observing that " $5^{2}$ has a remainder of 1 and 1 to any power is 1," combined also with John's comment ("This is an induction, right?"), seemed to have given them the conceptual insight that the remainder stayed 1 for all even numbers and some understanding into why this was the case. The students seemed to further understand the result when John stated: "If the first term $\left(5^{2 j}\right)$ has a remainder of 1 and $5^{2}$ has a remainder of 1 , can we assume their product has a remainder of 1 ?" While they are still not sure why their conjecture is true, this idea does convert into the technical handle needed to complete the inductive step of an induction proof, even 
though it took the students some time to operationally make this conversion.

Converting $5^{2 n}$ into $(3 k+1)$ led them to understand why their idea worked. Thus, the way this trio wrote the inductive step helped them make sense of this step, i.e., this step, combined with their conceptual insight, seemed to be making proving by induction explanatory for the students.

The students in the second trio started with more computations and made numerous algebraic mistakes. It was almost 20 minutes into the video before they started using induction. Once they came upon induction, they 'invented' their own approach to induction. In particular, they first assumed that $5^{2 j}$ had a remainder of 1 and $5^{2 j+1}$ had a reminder of 2 , and they then showed the statement for the next even $n$. What was interesting is that these students developed an induction argument unlike any they had previously seen and this induction approach convinced them that their conjecture was true. It appeared that the idea of using induction in this way arose naturally from their computations.

Several issues arose for each trio as they tried to write their induction proof. Both trios struggled with how to deal with division by 3 , initially writing $5^{2 n} / 3=3 i+1$. The first trio had the conceptual insight that the product of two numbers, each having a remainder of 1 when divided by 3 , should also have a remainder of 1 . Yet it took this trio a while to develop the technical handle to prove this. Both trios eventually were able to write the assumption of a remainder of 1 in the induction step as $(3 k+1)(3(8)+1)$, and were able to eventually prove that the expression with higher powers had a remainder 
of 1 for $n$ even and 2 for $n$ odd, with some minor errors (e.g., leaving out 'for some $k$ ' in their inductive step for one trio).

In terms of our framework, the problem was formulated such that induction was not an obvious method (aspect 1). The students in both trios used examples in their initial exploration of meaning (aspects 2 and 4) to construct a reasonable conjecture. They then developed the conceptual insight that the product of two numbers with a remainder of 1 should result in another number with a remainder of 1 . They turned this conceptual insight into the technical handle $(3 k+1)(3(8)+1)=3 j+1$ which then translated into an induction proof (aspect 3). The groups seemed to differ in their fluency with the necessary algebraic tools, but neither group had enough number theory experience to deal fluently with divisibility, such as using the form $3 k+1$ (aspect 3 ). As we described in some detail, the relation between students and the problem made it necessary to develop these technical handles, which they achieved through expressing algebraically the structures they had come to understand through exploration, eventually aligning their conceptual insights with the necessary technical means, concluding their proving activity with a proof that formalized their earlier exploration and that seemed to be explanatory for them.

What the students accomplished is close to 'cognitive unity' (Boero et al., 1996a), but their proof was not an organizing of some of their justifications. Instead, their explorations resulted in a conjecture - the product of two numbers with remainder one gives a number with remainder one - and a representation that helped them see induction as a useful proof method to apply in order to justify their conjecture. It is clear 
throughout that the goal for these students was to find and prove their conjecture. In doing so, the first group also developed a better knowledge of number theory and, in particular, an elementary result about modular arithmetic.

\section{Problem 2: Analysis of the Problem and Discussion of Students' Work}

\subsection{Analysis of Problem 2}

Problem 2: For what odd values of $n$ is the finite geometric series

$$
\sum_{i=0}^{n} 2^{i}
$$

a prime number? Prove your conjecture.

Like Problem 1, this problem formulation embodies the characteristics outlined in aspect 1 of the framework. Unlike Problem 1, it was more difficult for the students to discover the recursive nature of Problem 2. Superficially, this problem would not seem to relate to induction because, whether a particular odd integer $n$ results in a prime sum, it does not seem to relate to whether the next odd integer results in a prime sum. Also, in this problem students would likely need to construct examples to make a conjecture, which they can then try to prove (aspects 2 and 3).

The answer to the problem is that $n=1$ is the only odd integer such that the sum is prime because, in this case, the sum is 3 and for all other odd integers the sum is a composite divisible by 3 . The problem can be solved in many ways. One way would be for students to apply the sum recursively and use induction to show that the terms being added are always divisible by 3 . Another way would be for students to rewrite the sum as $2^{n+1}-1=2^{2 k}-1$ where $n=2 k-1$ and either use induction to prove that this 
expression is always divisible by 3 , or factor this expression as a difference of two squares. In any case, students need to convert a problem about primes to a problem about divisibility and, also, if they decide to use induction, they need to deal with the fact that the proof will be on the set of odd integers only.

\subsection{First trio's work on Problem 2}

This trio consisted of one female and two males, who we will call Alice, Bob, and Carl. After reading the problem, the students constructed the table below, adding the numbers incorrectly in the sums column.

\begin{tabular}{|l|l|}
\hline$n$ & $\sum_{i=0}^{n} 2^{i}$ \\
\hline 0 & \\
\hline 1 & 3 \\
\hline$z$ & 8 \\
\hline 3 & 16 \\
\hline 4 & 32 \\
\hline 5 & 64 \\
\hline
\end{tabular}

It appeared that the students were going to proceed with their calculation error.

Given the nature of the error and in the interest of time, the instructor recommended to the students that they check their work. As a result, they corrected their sums but, instead of continuing to re-write the results in a table, they used the following representation (presumably to ensure they were adding correctly): 


$$
\begin{array}{ll}
n=1: & 2^{0}+2^{1}=3 \\
n=3: & 2^{0}+2^{1}+2^{2}+2^{3}=15 \\
n=5: & 2^{0}+2^{1}+2^{2}+2^{3}+2^{4}+2^{5}=63 \\
n=7: & 2^{0}+2^{1}+2^{2}+2^{3}+2^{4}+2^{5}+2^{6}+2^{7}=255
\end{array}
$$

They quickly noticed then that 3 is the only prime and the other sums are composites divisible by 3. Bob said: "There might be another number later on if we continued, but I feel like there probably isn't so maybe we can try to find some sort of definition that says why $n=1$ is the only one where it would work." Carl agreed. There was a pause while they all looked at their work. At this point, even though they had the correct conclusion, they did not seem to have either a conceptual insight into why the statement is true or an idea of a technical handle to prove it.

They began a number of approaches to understand why the sums are always divisible by 3 , but each of these led nowhere and was abandoned after a few words. At some point Bob said, referring back to Carl: "There's got to be a reason why we're only taking into account the odd integers $n$. [pause] So that's got to have something to do with what you just said." After some more thinking, Carl said: "If we can conclude that they're always divisible by 3 , that would show that [pointing at the 3 ] is the only prime." After some thinking, Alice asked: "How can we show that?"

After another pause, Carl shifted to saying: "Well this, I guess we, why we add two numbers, like, what does that give us?" Alice: "What do you mean?" Carl: "Cause we're doing odds, so we're adding two sets of like [pointing at $2^{4}$ and $2^{5}$ ]." Bob then said: "Two to the next one." Bob's attention seemed to have shifted from looking at 
the sums to looking at the structure that arises from adding the powers of 2 . This shift turned out to be crucial because, after a short pause, Bob added: "We are always adding a number plus two times that number."

Carl: "Yes."

Bob: "Which is always divisible by 3. [pause] Think about it."

Alice: "Say it again."

At this point, Bob seemed to have developed a conceptual insight into why the sum is always divisible by 3 . Bob then pointed to a pair of added numbers. Carl then wrote " $+x+2 x=+3 x$ " and said: "If the original number is divisible by 3 and we always add $3 x$, it is always divisible by $3 . "$ This comment indicated that Carl, similarly to Bob, also seemed to have developed the same conceptual insight. The students then verbally went back through this argument, stating that the sum is three when $n=1$ and that each time you were adding a number divisible by 3 the sum remained divisible by 3. After this revisiting of their argument, there was a short pause. The instructor, conjecturing that the students hoped their explanation was sufficient, asked: "Can you prove it?" While this question might have been asked prematurely, it did spur the students into action, indicating they knew the difference between a heuristic argument and a proof. The students agreed that they could prove it because they had "the framework." Carl said: "Well, we understand it, it's just a matter of writing it out now" at which they all laughed.

The students first showed that the sum (=3) is prime when $n=1$. After looking at it in silence for a while, Carl said: "It sounds very much like an induction proof." From 
this point, it took them about 12 minutes to finalize the proof. We will not report this process in detail but there seemed to be two issues that the students faced in their work: (1) confusion because $n$ is only on the set of odd integers, so it took them some time to decide how to express odd numbers in their argument; and (2) difficulty expressing the argument in the form of a standard induction proof, partly because there was some disparity in what they knew (all the sums are divisible by 3 ) and what they were trying to prove ( $n=1$ results in the only prime sum). Eventually, though, the students were able to overcome both issues and complete the proof.

\subsection{Second trio's work on Problem 2}

This trio had two females and one male; we will call them Doris, Eve, and Fred. These students immediately wrote the following expression for the sum of the finite geometric series:

$$
\sum_{j=0}^{n} 2^{j}=\frac{1-2^{n+1}}{1-2}=2^{n+1}-1
$$

Doris stated that there is no formula for prime numbers and they rapidly set up the last three columns of the following table for odd values of $n$ (the students started completing the first column later, but left it incomplete).

\begin{tabular}{|l|l|l|l|l|}
\hline & $n$ & $2^{n+1}-1$ & & $\begin{array}{l}\text { [Added } \\
\text { later] }\end{array}$ \\
\hline $2(2)-1$ & 1 & 3 & $\mathrm{v}$ & $4-1$ \\
\hline $2(2)^{3}-1$ & 3 & 15 & $\mathbf{x}$ & $4^{2}-1$ \\
\hline & 5 & 64 & $\mathbf{x}$ & $4^{3}-1$ \\
\hline & 7 & 255 & $\mathbf{x}$ & $4^{4}-1$ \\
\hline
\end{tabular}




\begin{tabular}{|l|l|l|l|l|}
\hline & 9 & 1023 & $\times$ & $4^{5}-1$ \\
\hline
\end{tabular}

They had some brief sidetracks such as Eve wanting to generate more examples and Doris trying to solve

$$
2^{n+1}-1=13
$$

After about seven minutes of work on the problem, Fred began writing " $2(2)-1,2(2)^{3}-1$ " in the first column of the table and, looking for conceptual insight, said: "I'm trying to figure out why they are always divisible by 3." After a long pause, Doris said: "Uhmm, $2^{n+1}-1$. [pause] Would we use induction for that, show that if $2^{n+1}-1$. [pause] No, no, no, so that means $2^{n+1}-1$ equals $3 k$ because it's always divisible by 3 and then we would find..." Fred at that point came in with "what?" and then the trio proceeded to think about what $k$ equals, with Fred constructing the following table of finite differences between values of $k$ in $2^{n+1}-1=3 k$ :

\begin{tabular}{|l|l|l|l|l|l|l|l|}
\hline $\mathrm{k}=$ & 1 & & 5 & & 21 & & 85 \\
\hline differences & & 4 & & $4^{2}$ & & $4^{3}$ & \\
\hline
\end{tabular}

This was after about nine minutes of work on the problem. From here, the students dropped consideration of induction for nearly 17 minutes, making little progress on the problem. At this point, they added the last column in their original table and then looked again at Fred's table. Fred commented that he was trying to determine why the numbers in the added column, pointing at the $4^{j}-1$ terms, were always divisible by 3 . Over the next several minutes the trio tried a number of different 
approaches, including to rewrite $n=2 j+1$, but experienced confusion about the roles of $j$ and $n$, similar to that experienced by the first trio.

At about 42 minutes of work on the problem, the instructor suggested to the students they might want to revisit what it is they are trying to prove. In response to that, the students rewrote the problem as follows:

$$
\forall \mathrm{j} \in Z_{0}^{+}, \exists k \in Z \text { s.t. } 4^{j+1}-1=3 k \text {. }
$$

They then created the following table

\begin{tabular}{c|cccc}
$j$ & 0 & 1 & 2 & 3 \\
\hline$\frac{4^{j+1}-1}{3}$ & 1 & 5 & 21 & 85
\end{tabular}

which they noticed was the same as the table that Fred generated earlier. Unlike the first trio, however, these students had difficulty recognizing any process. With about 10 minutes left for filming, the instructor tried to help the students by asking if they could compute $k$ when $j=4$ without using a calculator. The students conjectured $k=4^{4}+85$ and, at this point, Fred said: “Ah, can we do induction with that? [pause] Maybe?” At this point, the students had discovered the conceptual insight that

$$
\frac{4^{j+1}-1}{3}=4^{j}+\text { previous number }
$$

which led them to the technical handle of induction.

The instructor then had a conversation with the students, pointing out they had mentioned induction almost 40 minutes earlier and then dropped it. He asked if they remembered that. The students laughed and said they did without, however, offering an 
explanation for why they abandoned the idea of induction. The instructor then asked if they could do the proof using induction, which they did, completing the proof in a few minutes, spending a total of 55 minutes on the problem. Their proof had a minor omission in that they used the inductive hypothesis that " $4^{j+1}-1=3 k$ for some $k$," without explicitly mentioning it in their argument.

\subsection{Commentary on both trios' work on Problem 2}

The problem formulation matched aspect 1 of the framework, as it did not suggest how to approach the problem; the problem was stated more like a problem a mathematician might be trying to answer while eliminating classes of numbers in searching for primes. As these students had experience in the utility of examples in proving (aspect 2 of the framework), they began by constructing examples to develop a conjecture. Through 'result process generalization' (Harel, 2001), both trios of students developed a conjecture that $n=1$ is the only case that is prime because all the other sums they computed are composites divisible by 3 . Relational necessity was also a characteristic of both trios' work with this problem, because the problem formulation was unfamiliar and so the students needed to construct and study examples to determine the underlying relation before they could proceed (aspect 4). As a result, both groups started their work on the problem not by trying to prove that all sums were

divisible by 3 , but by trying to develop conceptual insight into why this seemed to be the case based on the pattern they observed. This is a demonstration of aspect 2 of the framework, in that these students were constructing examples not just to verify but to expose structural relationships. 
Because of the addition error and apparently to ensure they were adding correctly, Alice, one of the students in the first trio, wrote out the sums in careful detail which allowed her group to notice a useful pattern. These students shifted to 'process pattern generalization' (Harel, 2001), generalizing the process they had discovered in going from one $n$-value to the next. The conjoining of their conceptual insight that they were adding terms divisible by 3 (these terms were the sum of $x$ and $2 x$ ) and their understanding of induction as a useful technical handle, together seemed to have explained for these students the reason their conjecture was true. In fact, the students said: "Well, we understand it, it's just a matter of writing it out." Also, we noticed that it was the formatting of their examples rather than the mere act of exemplification (the written sum instead of the table of values) that led to them seeing the underlying recursive structural relationship. Carl's pointing to the added terms $2^{4}$ and $2^{5}$ drew attention to the mathematical ideas embedded in the process by which $P(n)$ led to $P(n+2)$. This trio seemed to achieve cognitive unity since the students were able to identify and use a proof method, induction, that formalized justifications they offered during their exploratory work. Accordingly, for this trio proving by induction also served an explanatory function.

In contrast to the first trio's work on this problem, the form of the numbers $2^{n+1}$ -1 which the second trio rewrote as $4^{j+1}-1$, as seen in the last column of their first table, did not lead the second trio to looking at the process. There were clues this trio missed, such as Fred's noticing the same recursive pattern as the first trio did: that the difference of consecutive sums is a multiple of 3 , specifically $3\left(4^{i}\right)$ for some $i$. This 
observation led Doris to suggest induction, but her idea was not followed up in the group. If the trio had reflected on their first table, with the last column added in conjunction with Fred's table, they could have gained the conceptual insight that $\left(4^{2}-1\right)-\left(4^{1}-1\right)=3(4),\left(4^{3}-1\right)-\left(4^{2}-1\right)=3\left(4^{2}\right),\left(4^{4}-1\right)-\left(4^{3}-1\right)=$ $3\left(4^{3}\right)$. This conceptual insight can explain that the difference of consecutive sums is divisible by 3 and can lead to the algebraic technical handle necessary to convert this insight into a proof by induction, as it happened in the case of the first trio. The students in the second trio showed they were capable of doing this because, after the instructor asked them if they could compute $k$ when $j=4$ without using a calculator, they picked up the recursion and were able to write most of an induction proof. Why did this trio miss the recursive nature of this problem?

There are several plausible explanations for this question. One explanation relates to aspect 3 of the framework. The first trio's example space seemed to be sufficiently complex as to display the underlying recursive structural relationship. In addition, those students had sufficient proficiency with the technical handle of induction to recognize its use in this situation. On the other hand, the second trio did not think about looking for underlying relationships; they were looking more for patterns in the sums they could manipulate algebraically rather than for connections and insight. In addition, their proficiency with induction did not seem robust enough for them to continue using it when it occurred to them as a possibility at the early stages of their proving activity. Another explanation relates to aspect 2 of the framework. The first trio wrote the problem as what Harel (2001) calls a 'recursive problem.' Thus, when they 
were looking for why the sum is divisible by 3 , the form of their problem helped explain this. On the other hand, the second trio wrote the problem symbolically as what Harel (2001) calls a 'non-recursive problem,' and their symbolic format did not relate to the tables that offered the possibility of thinking recursively. When Fred said "I'm trying to figure out why they are always divisible by 3 " and Doris responded "Would we use induction for that ...", they proceeded by ignoring her idea. This relates to aspect 2 of the framework in that these students did not seem to have totally developed the habit of mind to construct examples to expose underlying structural relationships, so did not recognize the power of induction to understand why.

The first trio of students had their quasi-inductive argument together with an explanation, but it took some time for them to produce a proof, possibly because the problem involved two different concepts: prime numbers and divisibility. The operational obstacles did not seem to be cognitive: their earlier work on the problem indicated an understanding of the inductive step. Combining their comments and their written work it seems that their chosen representation provided a way for them to see through the process pattern to a conceptual insight about the inductive step, which then served as the technical handle for their proof. There was an alignment of the conceptual insight and the technical handle, through the extra terms added for each new sum, which allowed them to complete the proof (aspects 3 and 4 of the framework).

The second trio of students did not seem to have a conceptual insight into why all of the numbers were divisible by 3 , even though combining their tables could have 
explained it. However, all the instructor had to do to help them gain an insight was to ask the students to compute the next $k$-value without a calculator. The students even laughed about their dropping the idea of using induction after one of them suggested it. They later fairly quickly developed an almost correct induction proof after being prompted to prove the statement. They had some of the same operational difficulties in producing this proof as the first trio did, and probably for the same reasons.

To conclude, we can see from the first trio's investigation that, without explicitly thinking of induction, they developed a quasi-inductive argument to convince themselves of the truth of their conjecture and the reasons behind it, even though it took them some additional time to connect this to the proof method of induction. The proof by induction was then the codification of the conceptual insight and ideas they developed in their proving activity into why their conjecture was true. In other words, our analysis of the first trio's work provides an instance of how problem formulation, conjoined with a robust personal example space and a propensity to use examples to understand structural relationships, can result in a case in which the conceptual insight of recursive reasoning conjoins with the technical handle of proof by induction, resulting in a situation where proving by induction had explanatory power for the provers. Despite the second trio reformulating the problem, by way of their two tables, into a form that provided to them all the information they needed to discover that the change in the numbers is a multiple of 3 , this trio seemed to be stuck in working syntactically and did not look deeper into the structural relationship resulting from their examples (aspect 2 of the framework). As a result, they did not develop a conceptual insight into 
why the statement was true and the proving activity of these students did not serve an explanatory function.

\section{Overall Discussion of Students' Work on the Two Problems}

We now contrast the results of our analysis of students' work on the two problems with references to the four aspects of the framework. Both problems were posed in ways that did not directly imply using proof by induction, which was not the only possible proof method the students could use for their solution (aspect 1 of the framework). Also, none of the problems included an initial statement to prove, which means that the problems required exploration in students' proving activity.

Both problems led to exploration by exemplification, and the initial example spaces necessary for this were small positive integers, which were easily accessible to the students who had experience with the use of examples in proving (aspect 2). This led easily to students making conjectures about the problems. However, ways of expressing examples and their relevant properties leading to making sense of the underlying structure were not obvious and required some understanding of, and ways to represent, divisibility. Problem 1 required the relatively simple format $3 k+1$, which, although unfamiliar, eventually emerged. Problem 2 required a change of focus from primes to divisibility. In addition, the inductive step in Problem 2 was more complex, requiring the observation that the change in the sum each time was a multiple of $3-$ $x+2 x$ for the first trio and $3\left(4^{j}\right)$ for the second trio - which leads to the conceptual insight of the recursive nature of the problem. 
In terms of aspect 3 of the framework, students who have a stronger grounding in number theory might have expressed divisibility more quickly, but there would still be the extra step in Problem 2 of expressing the inductive step appropriately. There is therefore an interaction between the problem itself and the students' resources that we have described as 'relational necessity' in aspect 4 of the framework.

The first trios working each problem were more successful in moving towards a proof, and from this observation we conjecture that the explanatory potential of proving by induction might be realized if students alight on a way of representing and exploring the underlying ideas that give an explanation for the inductive step, possibly through the way they construct the process pattern generalization. For both problems helpful representation was present early on for the first trios but absent for the second trios. By 'helpful' we mean that the representation provides or expresses the conceptual insight in a recursive manner, which naturally led to the induction technical handle for those students.

For the second trios in each problem, students had more difficulty reasoning, interpreting, and manipulating their representations. The second trio working Problem 2 had the most difficulty. The first trio on Problem 2 looked at the structure of the problem, that is, how the numbers were changing, $x+2 x$. The students in the second trio working on this problem did recognize how the numbers were changing even though they discovered this relationship twice. They did not think about this relationship structurally, though, despite that this kind of thinking was within their reach (when the instructor asked for the next number, they were easily able to apply the pattern). The 
second trio therefore seemed to be locked into a syntactic mode and did not think about the structure, seeming to lack necessary proficiency in the utility of examples in proving (aspect 2). These difficulties of the second trios appear to arise from the fact that, although similarly to the other trios they were looking for conceptual insights for why the observed pattern was true, the form in which the second trios had represented the problems in their proving activity did not help them see how induction could serve an explanatory function for them.

\section{Conclusion}

When experienced mathematicians are given a statement to prove as an exercise, they might first ask themselves if there is a reasonable method to prove it and, if so, they may proceed with the method without necessarily having to think about why the statement is true. If stuck, then the mathematician may start exploring, trying to determine why the statement is true to see if they can get an insight into how to prove it. In other words, if a statement is relatively easy for the mathematician, it is likely to be worked in the proving activity as if it were a procedure before reflecting on the implications; if it is more complex, it becomes a problem displaying some relational necessity to explore in more depth.

In the cases we discussed in this paper, we tried to implement a similar idea with undergraduate mathematics students. We hypothesized that if students are working on problems that do not clearly offer in their formulation the statement they need to prove, then students would likely engage in exploring examples and possible 
relationships. Given students' experience with the use of examples to explore structure when proving, this exploration could, in turn, lead them to construct informally the inductive step and, eventually, see the utility and apply mathematical induction in their proving activity to formalize their work. In such situations, proving by mathematical induction becomes a codification of students' sense making and serves first as a method that explains and then as a proof that verifies.

Our hypothesis found support in the work of both trios on Problem 1 and the first trio on Problem 2. The work of these trios involved problem formulations not straightforwardly asking for a proof, the students had experience with the utility of examples in proving, and the students were familiar (but not proficient) with induction and were expected to be able to recognize/apply it as an appropriate proof method in their explorations, thus satisfying the four aspects of our framework. Where there were weaknesses, they did not seem to be in students' understanding of mathematical induction but in formulating an appropriate expression of the ideas in the statement during the students' initial exploration of the problem. It is important, of course, that the prover has the technical expertise to construct, manipulate, and make sense of suitable expressions, and this might be improved by a focused study of number theory so that suitable expressions and techniques were more readily available to be used. However, in this study students were given the time they needed to explore and to make an appropriate use of their existing technical expertise and so they were able to make some progress in relation to number theory as well as in their experience of using induction. 
Going back to our discussion of Smith's (2006) study, she reported on a student who was uncomfortable with induction because she looked for "arguments that explain why processes work" (p. 81, emphasis in original). Similarly to experienced mathematicians, we hope that, with practice, students will learn to consider induction when first looking for a reasonable method to use in their proving activity. The analysis of the three trios who used induction in a way that had an explanatory power for them illustrates the success students can have when given an appropriately phrased problem (aspect 1), provided also that the students have necessary qualities to benefit from engaging with such a problem (aspects 2 and 3). The second trio's work on Problem 2 illustrates that, even when the four aspects of the framework may be satisfied, the students may not experience the explanatory power of proving by induction unless they alight on a suitable expression. While Lange (2009) argued that proof by induction is never explanatory, our findings suggest that proving by induction can be explanatory, at least in the subjective perspective we adopted in this paper, and the satisfaction of the four aspects of our framework can enhance the likeliness of this happening for the prover.

\section{References}

Bell, A.W. (1976). A study of pupil's proof-explanations in mathematical situations. Educational Studies in Mathematics, 7(1), 23-40.

Boero, P., Garuti, R., Lemut, E., \& Mariotti, M. A. (1996a). Challenging the traditional school approach to theorems: A hypothesis about the cognitive unity of 
theorems. In L. Puig \& A. Gutiérrez (Eds.), Proceedings of the 20th Conference of the International Group for the Psychology of Mathematics Education (Vol. 2, pp. 113-120). Valencia, Spain: University of Valencia.

Boero, P., Garuti, R., \& Mariotti, M. A. (1996b). Some dynamic mental processes underlying producing and proving conjectures. In L. Puig \& A. Gutiérrez (Eds.), Proceedings of the 20th Conference of the International Group for the Psychology of Mathematics Education (Vol. 2, pp. 121-128). Valencia, Spain: University of Valencia. Brown, S. (2008). Exploring epistemological obstacles to the development of mathematics induction. In M. Zandieh (Ed.) Proceedings of the $11^{\text {th }}$ Conference for Research on Undergraduate Mathematics Education. Published electronically at http://sigmaa.maa.org/rume/crume2008/Proceedings/S_Brown_LONG.pdf.

Cariani, F. (2011) Mathematical induction and explanatory value in mathematics. Available electronically at http://cariani.org/files/MIEV.cariani.pdf

Davis, M., Grassl, R., Hauk, S., Mendoza-Spencer, B., \& Yestness, N. (2009). Learning proof by mathematical induction. In M. Zandieh (Ed.), Proceedings of the $12^{\text {th }}$ Conference on Research in Undergraduate Mathematics Education. Published electronically at http://sigmaa.maa.org/rume/crume2009/MDavis_LONG.pdf. de Villiers, M. (1999). The role and function of proof. In M. de Villiers (Ed.), Rethinking proof with the Geometer's Sketchpad (pp. 3-10). Key Curriculum Press.

Dubinsky, E. (1986). Teaching mathematical induction I. Journal of Mathematical Behavior, 5, 305-317. 
Dubinsky, E. (1990). Teaching mathematical induction II. Journal of Mathematical Behavior, 8(3), 285-304.

Dubinsky, E., \& Lewin, P. (1986). Reflective abstraction and mathematics education: The generic decomposition of induction and compactness. Journal of Mathematical Behavior, 5(1), 55-92.

Hanna, G. (1990). Some pedagogical aspects of proof. Interchange, 21(1), 6-13. Hanna, G. (2000). Proof, explanation and exploration: An overview. Educational Studies in Mathematics, 44, 5-23.

Garuti, R., Boero, P., \& Lemut, E. (1998). Cognitive unity of theorems and difficulty of proof. In A. Olivier \& K. Newstead (Eds.), Proceedings of the 22nd Conference of the International Group for the Psychology of Mathematics Education (Vol. 2, pp. 345-352). Stellenbosch, South Africa: University of Stellenbosch. Harel, G. (2001). The development of mathematical induction as a proof scheme: A model for DNR-based instruction. In S. Campbell \& R. Zazkis (Eds.), Learning and teaching number theory: Research in cognition and instruction (pp. 185-212). Dordrecht, The Netherlands: Kluwer.

Harel, G., \& Sowder, L. (1998). Students' proof schemes: Results from exploratory studies. In A. H. Schoenfeld, J. Kaput, \& E. Dubinsky (Eds.), Research in collegiate mathematics education III (pp. 234-283). Providence, RI: American Mathematical Society.

Harel, G., \& Sowder, L. (2007). Toward comprehensive perspectives on the learning and teaching of proof. In F. K. Lester (Ed.), Second Handbook of Research on 
Mathematics Teaching and Learning (pp. 805-842). Greenwich, CT: Information Age Publishing.

Hersh, R. (1993). Proving is convincing and explaining. Educational Studies in Mathematics, 24(4), 389-399.

Kitcher, P. (1989). Explanatory unification and the causal structure of the world. In P. Kitcher and W.E. Salmon (Eds.), Scientific explanation (pp. 410-505). Minneapolis: University of Minnesota Press.

Knuth, E.J. (2002). Secondary school mathematics teachers' conceptions of proof. Journal for Research in Mathematics Education, 33(5), 379-405.

Lange, M. (2009). Why proofs by mathematical induction are generally not explanatory. Analysis, 69, 203-211.

Maher, C., \& Martino, A. (1996). The development of the idea of mathematical proof: A 5-year case study. Journal for Research in Mathematics Education, 27, 194-214. Maher, C., Muter, E., \& Kiczek, R. (2007). The development of proof making by students. In P. Boero (Ed.), Theorems and proof in schools: From history, epistemology and cognition to classroom practice (pp. 197-208). Rotterdam: Sense Publishers.

Mariotti, M. A. (2006). Proof and proving in mathematics education. In A. Gutiérrez \& P. Boero (Eds.), Handbook of research on the PME: Past, present and future (pp. 173-204). Rotterdam, The Netherlands: Sense.

Movshovitz-Hadar, N. (1993). The false coin problem, mathematical induction and knowledge fragility. Journal of Mathematical Behavior, 12(3), 253-268. 
Raman, M. (2003). Key ideas: What are they and how can they help us understand how people view proof? Educational Studies in Mathematics, 52, 319-325. Raman, M., Sandefur, J., Birky, G., Campbell, C., \& Somers, K. (2009). Is that a proof? Using video to teach and learn how to prove at the university level. In F.L. Lin, F.J. Hsieh, G. Hanna, \& M. de Villiers (Eds.), Proceedings of ICMI Study 19 on Proof and Proving in Mathematics Education (vol. 2, 154-159). Taipei: National Taiwan Normal University.

Reid, D. (2002). Conjectures and refutations in grade 5 mathematics. Journal for Research in Mathematics Education, 33(1), 5-29.

Resnik, M., \& Kushner, D. (1987). Explanation, independence, and realism in mathematics. British Journal for the Philosophy of Science, 38(2), 141-158.

Ron, G., \& Dreyfus, T. (2004). The use of models in teaching proof by mathematical induction. Proceedings of the $28^{\text {th }}$ Conference of the International Group for the Psychology of Mathematics Education (vol. 4, pp. 113-120). Bergen, Norway.

Sandefur, J., Mason, J., Stylianides, G.J., \& Watson, A. (2013). Generating and using examples in the proving process. Educational Studies in Mathematics, 83(3), 323340.

Smith, J.C. (2006). A sense-making approach to proof: Strategies of students in traditional and problem-based number theory courses. Journal of Mathematical Behavior, 25, 73-90.

Steiner, M. (1978). Mathematical explanation. Philosophical Studies, 34(2), 135151. 
Stylianides, A.J. (2007). Proof and proving in school mathematics. Journal for Research in Mathematics Education, 38, 289-321.

Stylianides, G.J. (2009). Reasoning-and-proving in school mathematics textbooks. Mathematical Thinking and Learning, 11, 258-288.

Stylianides, G.J., \& Stylianides, A.J. (2009). Facilitating the transition from empirical arguments to proof. Journal for Research in Mathematics Education. 40, 314352.

Stylianides, G.J., Stylianides, A.J., \& Philippou, G. N. (2007). Preservice teachers' knowledge of proof by mathematical induction. Journal of Mathematics Teacher Education, 10, 145-166.

Stylianides, G.J., Stylianides, A.J., \& Weber, K. (2016). Research on the teaching and learning of proof: Taking stock and moving forward. In J. Cai (Ed.), First Compendium for Research in Mathematics Education. Reston, VA: National Council of Teachers of Mathematics. (forthcoming)

Weber, K. (2010). Proofs that develop insight. For the learning of mathematics, $30(1) 32-36$. 